\title{
Capacity of a Relay Acoustic Channel
}

\author{
Milica Stojanovic \\ Massachusetts Institute of Technology \\ millitsa@mit.edu
}

\begin{abstract}
A relay acoustic link consisting of $N$ hops spanning a fixed distance $d$ is considered. The capacity of the relay link is found to increase with the number of hops as $N^{\gamma}$, where $\gamma$ is a positive constant less than 1 . At the same time, the power required to span the link decreases, but because each additional relay introduces a fixed cost, the number of relays in a practical system is limited. Taking this fact into account, an overall cost is defined, based either on a power, or an energy per bit criterion. Minimization of the cost function provides an analytical solution for the optimal number of relays.

The cost of relaying is also evident in the delay, as each relay introduces a delay proportional to the data packet length. However, the distance between relays becomes shorter as more relays are added, thus supporting transmission at a higher rate. Assuming transmission at a rate equal to the channel capacity, the overall link delay is found to behave as $N^{1-\gamma}$. Compared to a situation in which transmission rate is chosen irrespective of the number of relays (a linear increase of the delay with $N$ ), rate adjustment offers an improved delay performance.
\end{abstract}

\section{INTRODUCTION}

It is well known that the bandwidth of an underwater acoustic communication system is severely limited. Moreover, since the path loss in an acoustic channel depends both on the distance and on the frequency of the signal, the bandwidth, as well as the power required to achieve a pre-specified signal-to-noise ratio (SNR), depends on the link distance. This dependence was assessed analytically in [1]. In particular, it was shown that the bandwidth can be approximated as $B(d)=b d^{-\beta}$, where $d$ is the transmission distance, and $b$ and $\beta$ are positive constants. Similar relationships were found to hold for the link capacity and the transmission power.

In this paper, the results of [1] are extended, and applied to a relay acoustic channel. A communication scenario is considered in which a certain number of bits have to be transmitted over a distance $d$. This task can be accomplished in a single hop, or by dividing the link into $N$ hops, each of length $d / N$. In the multi-hop case, a relay acoustic node is employed at each hop. The relay nodes receive the signal, regenerate it, and pass it on to the next hop, until the final destination is reached. Shorter links require less transmission power and at the same time offer a greater bandwidth, thus motivating the multi-hop approach. The question of interest to a system designer is how exactly do the two approaches compare in terms of the link capacity, total power consumed, energy per bit, overall cost and delay. Given a particular optimization criterion, the question also arises as to whether there exists an optimal number of hops to use over a given link.
To answer these questions, we take an information-theoretic point of view, which assumes that transmission over each hop can be accomplished at a rate equal to that hop's capacity. The capacity of a relay channel is thus equal to the capacity of each of its hops, and is shown to increase with the number of relays. Since relaying also helps to reduce the total transmission power, its benefits on an acoustic link are even more pronounced in view of the energy per bit savings.

In practice, however, one cannot count on using a large number of relays, as each relay adds to the deployment cost. To take this fact into account, an overall system cost is defined, for example as the total transmission power needed to span the link, plus a fixed deployment cost per relay. Alternatively, the cost can be based on an energy per bit criterion. Delay may be viewed as yet another form of cost associated with relaying.

In this paper, we consider system optimization in light of minimizing an associated cost function. The results quantify advantages of multi-hopping along an acoustic link, and emphasize the benefits of a system design in which transmission rate is optimized according to the number of hops. These results make use of a basic acoustic path loss model, thus providing a guideline for the design of a general relay acoustic link. The analysis can be extended to include a more complicated, site-specific propagation model.

The paper is organized as follows. In Sec.II, the capacitydistance relationship [1] is reviewed, and a model is established for the bandwidth, capacity and power needed to achieve a desired SNR. Sec.III is devoted to the capacity analysis of the relay link. System optimization based on the power and energy per bit cost functions is addressed in In Sec.IV. The issues of delay are considered in Sec.V. Numerical examples are used throughout the discussion to illustrate the results, and the conclusions are summarized in Sec.VI.

\section{SySTEM MODEL}

A simple mathematical model [1] was developed to assess the dependence of an acoustic communication channel capacity on the distance. To do so, the acoustic path loss experienced by a signal of frequency $f$ traveling over a distance $x$ was modeled as $A(x, f) \sim x^{k} \cdot a(f)^{x}$, where $k$ is the spreading factor and $a(f)$ the absorption coefficient. Accompanying the capacity calculations were two types of bandwidth selection: one based on a heuristic, $3 \mathrm{~dB}$ design principle, and another on an optimal, capacity-maximizing principle. With each type of bandwidth selection, there is an associated transmission power required to achieve a pre-specified SNR at the receiver. 
Numerical evaluation of the analytical results of [1] indicated the possibility to obtain closed-form semi-analytical solutions for the link capacity $C$, bandwidth $B$, and transmission power $P$ as functions of distance. In particular, it was shown that for a given required SNR, the following relationships hold:

$$
\begin{aligned}
& B(x)=B_{0}\left(x / x_{0}\right)^{-\beta}=b x^{-\beta} \\
& C(x)=C_{0}\left(x / x_{0}\right)^{-\gamma}=c x^{-\gamma} \\
& P(x)=P_{0}\left(x / x_{0}\right)^{\psi}=p x^{\psi}
\end{aligned}
$$

Each of these quantities is thus characterized by two model parameters: the scaling factor and the exponent ( $b$ and $\beta$ for the bandwidth, respectively). The model parameters are obtained by a first-order least-squares polynomial approximation on a logarithmic scale. These parameters were evaluated in [1] for the design SNR of $20 \mathrm{~dB}$, and comparison with the true values demonstrated an excellent match.

Here, we extend the results of [1] to assess the dependence of the model parameters on the SNR. For each target SNR, $\mathrm{SNR}_{0}$, semi-analytical solutions are obtained, and the two model parameters (the scaling factor and the exponent) are evaluated. We do this for both the optimal and the heuristic 3 $\mathrm{dB}$ bandwidth selection, labeling the first one by the subscript $o$ and the second one by the subscript 3. In other words, the model that we propose for the optimal system bandwidth is of the form $B_{o}\left(x, S N R_{0}\right)=b_{o}\left(S N R_{0}\right) x^{-\beta_{o}\left(S N R_{0}\right)}$. The capacity and the power are modeled in a similar manner.

Figs.1-4 illustrate the model parameters as functions of the SNR. These results were obtained using the spreading loss corresponding to a practical value $k=1.5$, and the ambient noise power spectral density corresponding to calm seas and a moderate shipping activity as in [1]. Figs.1 and 2 refer to the optimal bandwidth definition, while Figs. 3 and 4 refer to the $3 \mathrm{~dB}$ bandwidth definition. In both cases, the coefficients are given in $\mathrm{dB}$ relative to $1 \mathrm{kHz}, 1 \mathrm{kbps}$, and 1 $\mu \mathrm{Pa}$, for the bandwidth, capacity, and power, respectively, and the exponents are given in $\mathrm{dB}$ per $\mathrm{km}$.

The results indicate that the bandwidth and capacity exponents are practically the same under each definition, $\beta_{o} \approx \gamma_{o}$ and $\beta_{3} \approx \gamma_{3}$. The exponents $\beta_{3}, \gamma_{3}, \psi_{3}$ are invariant to the SNR, while the exponents $\beta_{o}, \gamma_{o}, \psi_{o}$ exhibit some SNR dependence. All the scaling factors are increasing functions of the SNR, except for $b_{3}$ which is constant. We note that it would also be possible to provide closed-form functional dependences of the model parameters on the SNR by further approximations (for example, the scaling factor $p_{3}$ is seen to follow a linear trend on the logarithmic scale).

In the following section, we will use the bandwidth, capacity and power models to analyze a simple relay acoustic link. Note that these models may find a broader use in the design and analysis of an arbitrary underwater network, as they offer a quick and easy way of calculating the system resources that need to be allocated for a given network topology. This fact in turn enables a computationally affordable analysis of various network topologies, which may be needed as part of a general network optimization.
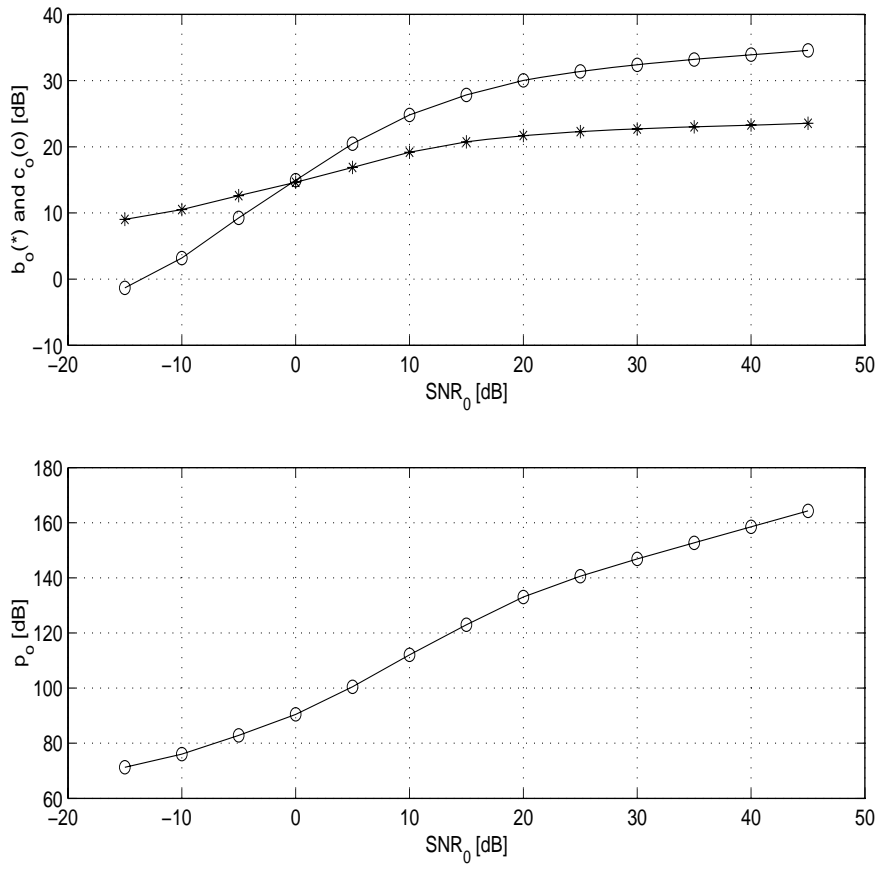

Fig. 1. Model parameters as functions of target SNR under the capacitymaximizing definition: bandwidth and capacity scaling factors $b_{o}, c_{o}$, and power scaling factor $p_{o}$.

\section{CAPACITY ANALYSIS}

We consider a relay link as shown in Fig.5. The distance $d$ is divided into $N$ hops, such that the length of each hop is $d / N$. The link is designed so that the SNR at the input to each relay equals the target value $\mathrm{SNR}_{0}$. This design ensures a fair comparison between a multi-hop and a single-hop link. In what follows, we shall use the capacity in the optimal sense, i.e. the bandwidth and the power will be defined as those that maximize the channel capacity under the constraint of fixed total power needed to ensure the desired SNR.

Using the expressions (1), the capacity of each hop is determined as $C(d / N)$, and the capacity of the relay link is equal to

$$
C_{N}(d)=C(d / N)
$$

A similar relationship can be established for the bandwidth,

$$
B_{N}(d)=B(d / N)
$$

Fig.6 illustrates the capacity of the relay link. The capacity $C_{N}(d)$ is shown versus the number of hops $N$ for varying link distance $d$. We observe that the capacity increases with the number of hops for a given link distance. As expected, the capacity is lower for a greater link distance. The bandwidth $B_{N}(d)$ follows a similar trend, as shown in Fig.7.

The bandwidth efficiency, defined as $C_{N}(d) / B_{N}(d)$, is shown in Fig.8. It follows a reverse trend, decreasing as the hop distance becomes shorter. However, we note that the absolute value of the bandwidth efficiency does not change much for the range of values $d$ and $N$ shown. 

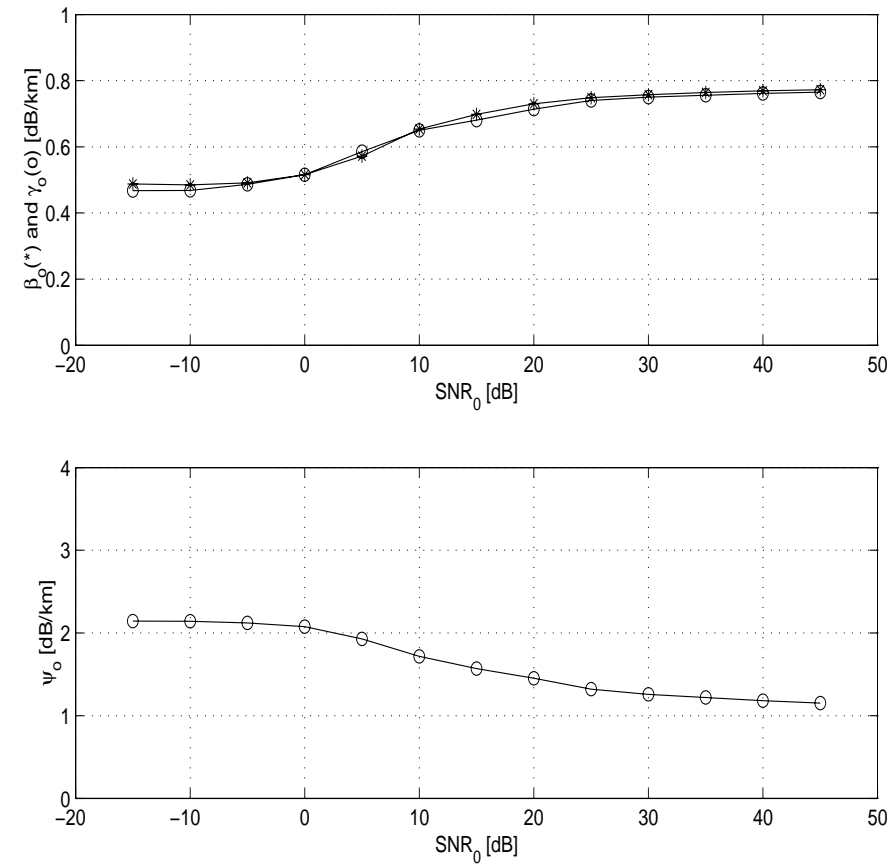

Fig. 2. Model parameters as functions of target SNR under the capacitymaximizing definition: bandwidth and capacity exponents $\beta_{o}, \gamma_{o}$, and power exponent $\psi_{o}$.

The power needed to transmit over one hop is equal to $P(d / N)$, and the power needed to span the entire link is

$$
P_{N}(d)=N P(d / N)
$$

This power is shown in Fig.9. As it can be expected, less power is required for a greater number of relays. While such a situation is typical of any wireless channel, the heavy dependence of the capacity on the number of relays is particular to the underwater acoustic channel. This fact bears influence on the energy per bit needed to transmit over the distance $d$. If transmission is accomplished over $N$ hops, the energy per bit is

$$
E_{N}(d)=\frac{P_{N}(d)}{C_{N}(d)}=\frac{N P(d / N)}{C(d / N)}
$$

Fig.10 shows how the energy $E_{N}(d)$ decreases with the number of hops. The benefits of multi-hopping are most evident in this metric, as it combines the power reduction and the capacity (bandwidth) increase which occur simultaneously as the given link is divided into more hops.

\section{System Optimization}

Our treatment so far indicates that the link capacity, as well as the energy consumption of a multi-hop acoustic link improve with the number of relays. While it may appear that the best design is one that uses the greatest number of hops, the number of relays in a practical system cannot be increased indefinitely, as each relay introduces additional costs. These costs can be viewed in light of the actual hardware and deployment costs, and also in light of the total delay.
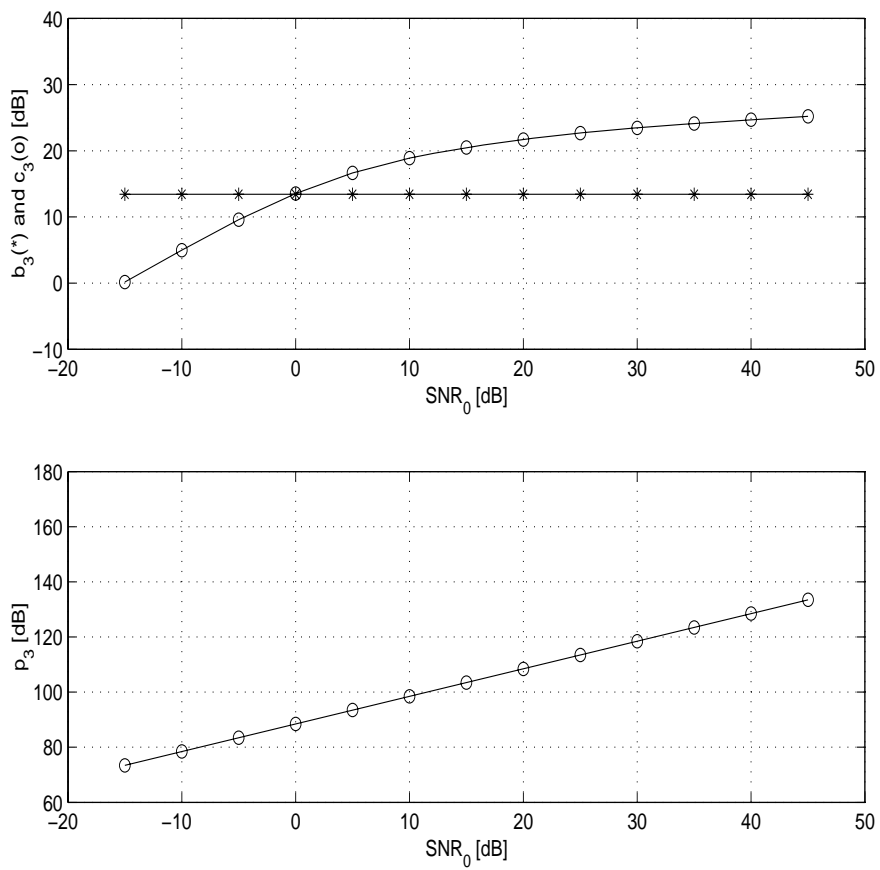

Fig. 3. Model parameters as functions of target SNR under the $3 \mathrm{~dB}$ definition: bandwidth and capacity scaling factors $b_{3}, c_{3}$, and power scaling factor $p_{3}$.

In this section, we focus on two performance measures, one based on the total power consumption to which fixed deployment costs are added, and another based on the energy per bit needed to span the link. In each case, we address the impact of the number of hops on the system performance as defined by the appropriate cost function.

Taking the total power consumption as the base for evaluating the system cost, let us define the cost function as

$$
K(N)=P_{N}(d)+(N-1) K_{0}
$$

where $P_{N}(d)$ is the power needed to transmit over $N$ hops (4) and $K_{0}$ is the fixed per-node deployment cost. Fig.11 illustrates the cost function versus the number of hops. This example clearly shows the existence of an optimal number of hops to use over a given link distance $d$. For instance, $N=6$ hops ( 5 relays) is the choice that minimizes the cost function for a $30 \mathrm{~km}$ link. At the same per-node deployment cost, a 20 km link is best spanned in $N=4$ hops.

The optimal number of hops can also be determined analytically, by differentiating the cost function with respect to $N$, and setting the derivative equal to zero. Using the model (1) for the power, and treating $N$ as a continuous variable, the following result is obtained: ${ }^{1}$

$$
\tilde{N}=d\left[\frac{p(\psi-1)}{K_{0}}\right]^{1 / \psi}
$$

Hence, the optimal number of hops under the power-based cost function is proportional to the link distance.

\footnotetext{
${ }^{1}$ Note from Fig. 2 that $\psi>1$.
} 

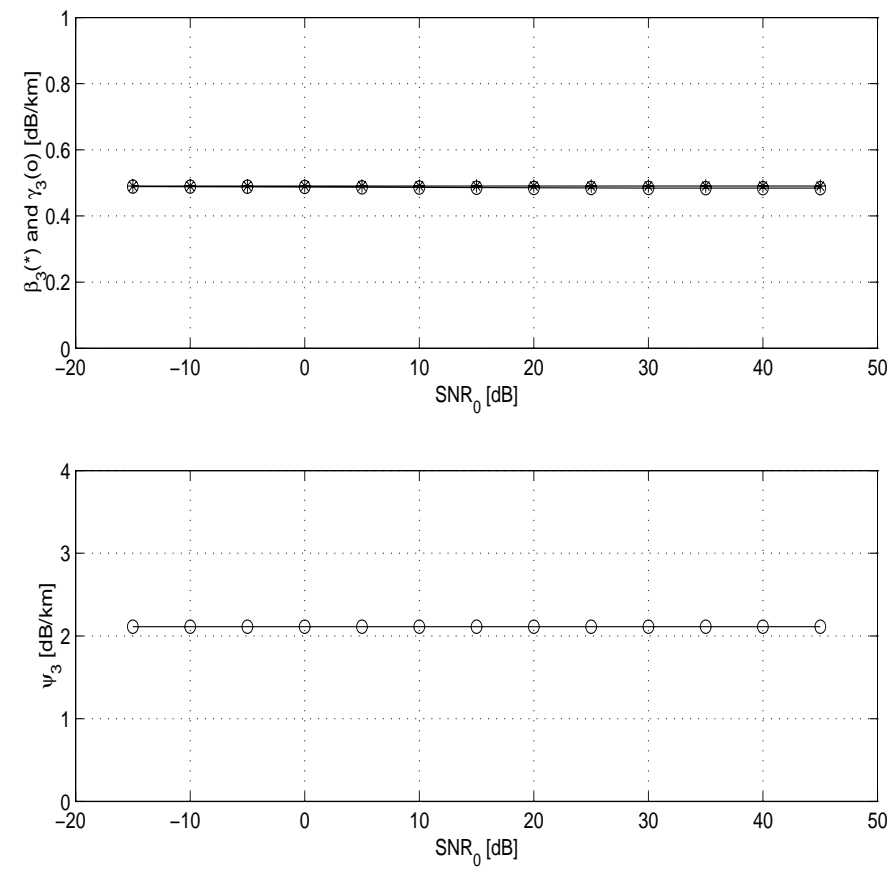

Fig. 4. Model parameters as functions of target SNR under the $3 \mathrm{~dB}$ definition: bandwidth and capacity exponents $\beta_{3}, \gamma_{3}$, and power exponent $\psi_{3}$.

Fig.12 shows the optimal number of hops, taken as the integer value $\lceil\tilde{N}\rceil$, for the example considered. Given the system model (1), it is straightforward to generate results for a different deployment geometry.

It is also possible to define a cost function based on the energy per bit, rather than the total power consumption. Such a cost functions is defined as

$$
K^{\prime}(N)=E_{N}(d)+(N-1) K_{0}^{\prime}
$$

where $K_{0}^{\prime}$ is a fixed per-node cost. Fig.13 illustrates this cost function, which exhibits a minimum, i.e. demonstrates the existence of an optimal number of hops to use over a given acoustic relay link. The optimal number of hops under the energy-based cost function can also be obtained in the closed form. The solution is in the same form as that given by the expression (7), with $(\psi+\gamma)$ in place of $\psi$, and $K_{0}^{\prime}$ in place of $K_{0}$. The question that remains open, or rather left for the practitioners to address, is how to set the value of the fixed per-node deployment cost.

\section{Delay}

Total transmission delay is another important metric in the design of a relay link. In a digital communication system, it is beneficial that relays regenerate a packet, rather than simply amplifying and forwarding it. By doing so, noise accumulation along the link is prevented, and the performance, as measured by the overall bit error rate (BER), stays close to that of a single-hop link with the same SNR. In particular, the BER of an $N$-hop link is given by

$$
P_{e, N}\left(\mathrm{SNR}_{0}\right)=1-\left(1-P_{e, 1}\left(\mathrm{SNR}_{0}\right)\right)^{N} \approx N P_{e, 1}\left(\mathrm{SNR}_{0}\right)
$$

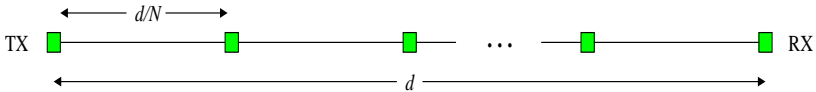

Fig. 5. The relay link.

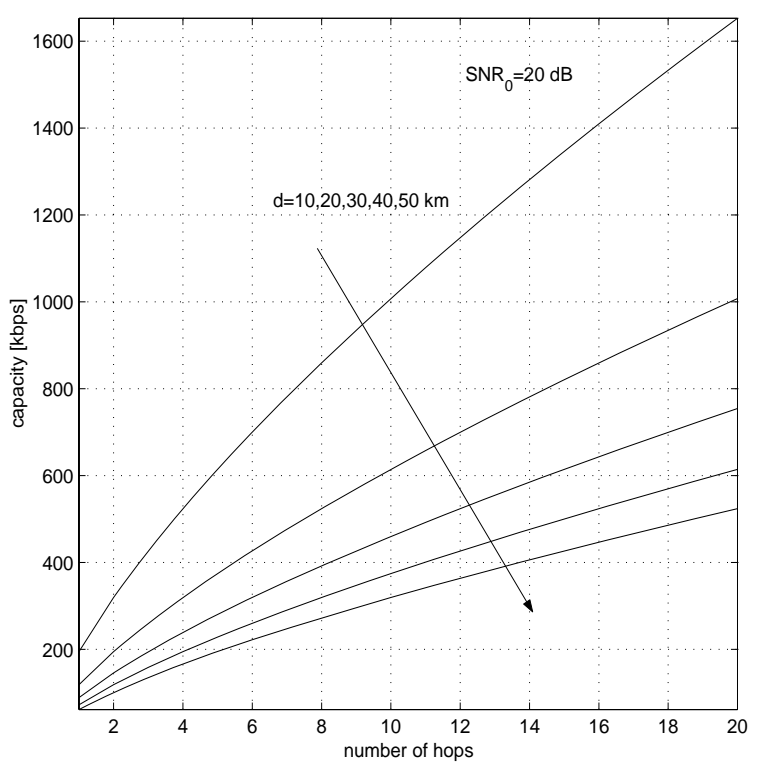

Fig. 6. Capacity of the relay channel as a function of the number of hops $N$, for varying link distance $d$.

where $\mathrm{SNR}_{0}$ is the SNR at the input to each relay, and $P_{e, 1}$ is the BER of each hop. The approximation normally holds, since the link is designed such that $P_{e, 1}\left(\mathrm{SNR}_{0}\right)<<1$. Hence, the overall BER $P_{e, N}$ is only scaled, which is a minor degradation for a practical number of hops. However, the process of regeneration requires that the entire packet be received before it is passed on to the next relay, which introduces a delay.

In addition to forward error correction, regeneration allows for automatic repeat request (ARQ) of packets received in error. In what follows, we focus for simplicity on the basic delay that occurs due to regeneration, not taking into account the time spent in repeated transmissions. The delay/throughput analysis of the ARQ data link is deferred to a later publication. 


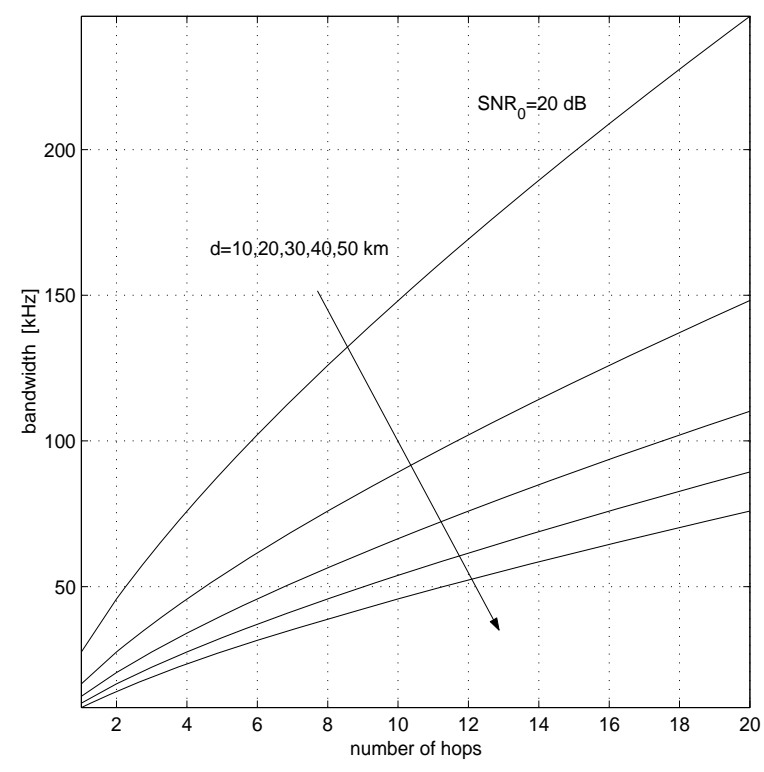

Fig. 7. Bandwidth of the relay channel as a function of the number of hops $N$, for varying link distance $d$.

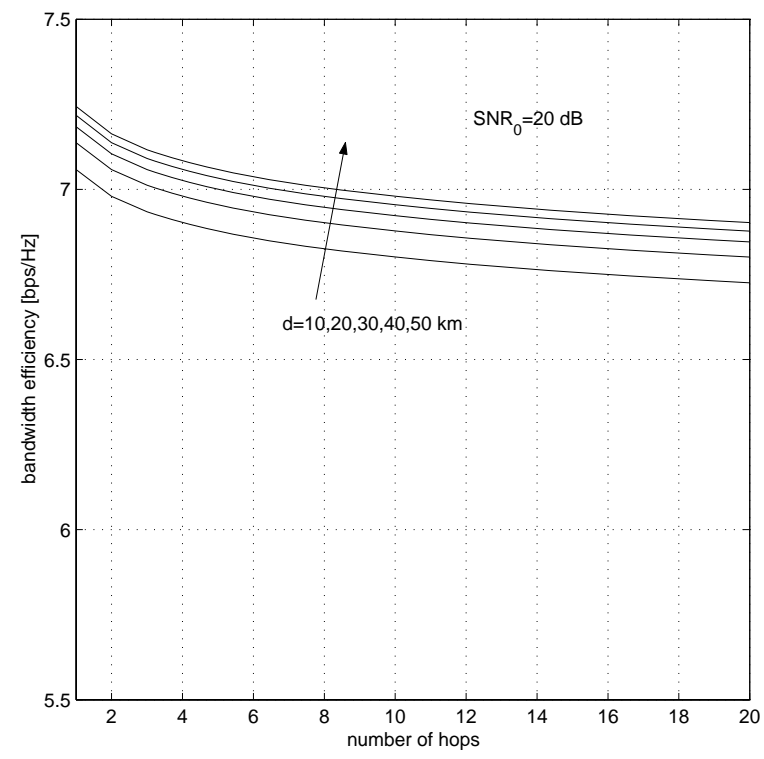

Fig. 8. Bandwidth efficiency of the relay channel as a function of the number of hops $N$, for varying link distance $d$.

Fig.14 illustrates the delay incurred in regenerating a packet at the $n$th relay node. If a packet contains $L$ bits (binary modulation can be assumed without the loss of generality) its duration is $T_{L}=L / R$, where $R$ is the bit rate. Between each two relays separated by a distance $d / N$, there is a propagation delay of $d / N c_{s}$ seconds, where $c_{s}$ is the speed of sound underwater (nominally $1500 \mathrm{~m} / \mathrm{s}$ ). Packet regeneration introduces an additional delay equal to the packet duration $T_{L}$. Hence, the per-hop delay is $T_{L}+d / N c_{s}$, and the total delay that a packet experiences over $N$ hops is

$$
D_{N}(d)=N\left[\frac{d}{N c_{s}}+\frac{L}{R}\right]=\frac{d}{c_{s}}+\frac{L}{R} N
$$

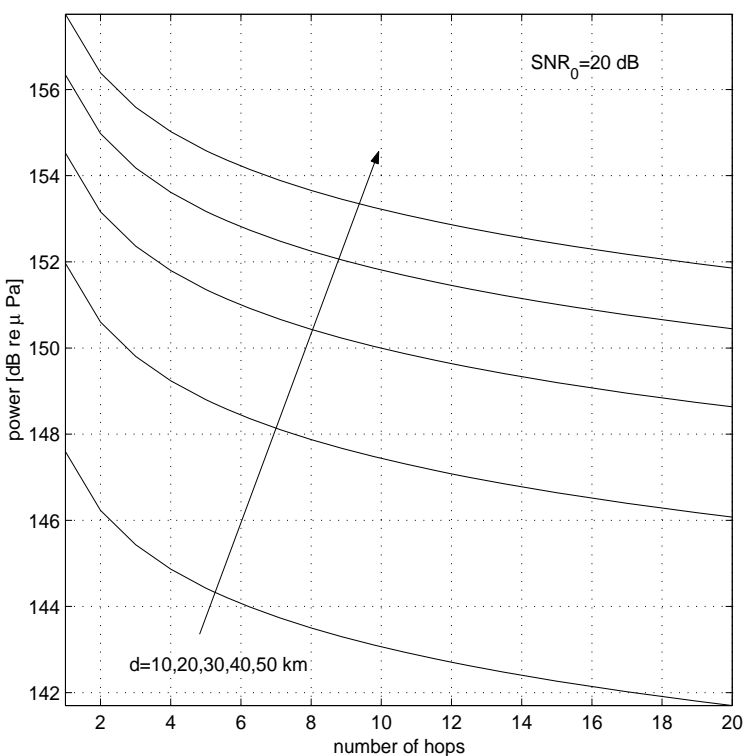

Fig. 9. Total transmission power vs. the number of hops $N$. The power is determined so as to ensure $20 \mathrm{~dB}$ of SNR at the input to each relay.

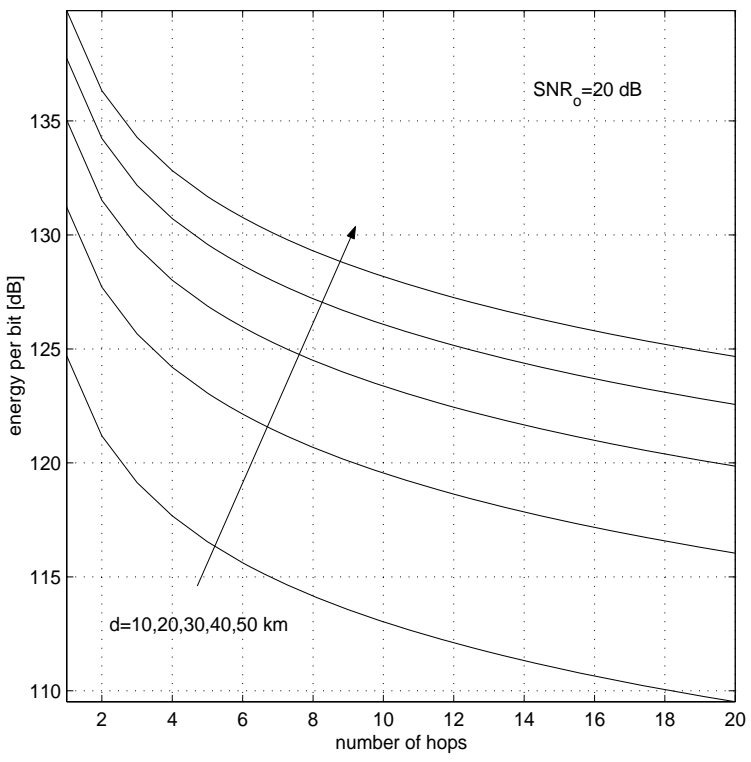

Fig. 10. Energy per bit need for relaying over $N$ hops.

The overall delay thus increases with $N$, and this fact may impose a limit on the number of relays to be used in a given system.

To assess the effect of the number of relays on the overall delay, we will study two cases. In the first case, the transmission rate $R$ is fixed regardless of the number of hops. The delay in this case is linearly proportional to the number of relay nodes. This situation would occur in a system that uses existing off-the-shelf acoustic modems, which currently offer a limited range of pre-determined, fixed bit rates. For example, the WHOI Micro-Modem currently offers two regimes of operation at $80 \mathrm{bits} / \mathrm{s}$ or $5000 \mathrm{bits} / \mathrm{s}$, while commercially available modems offer similar ranges [2]. 


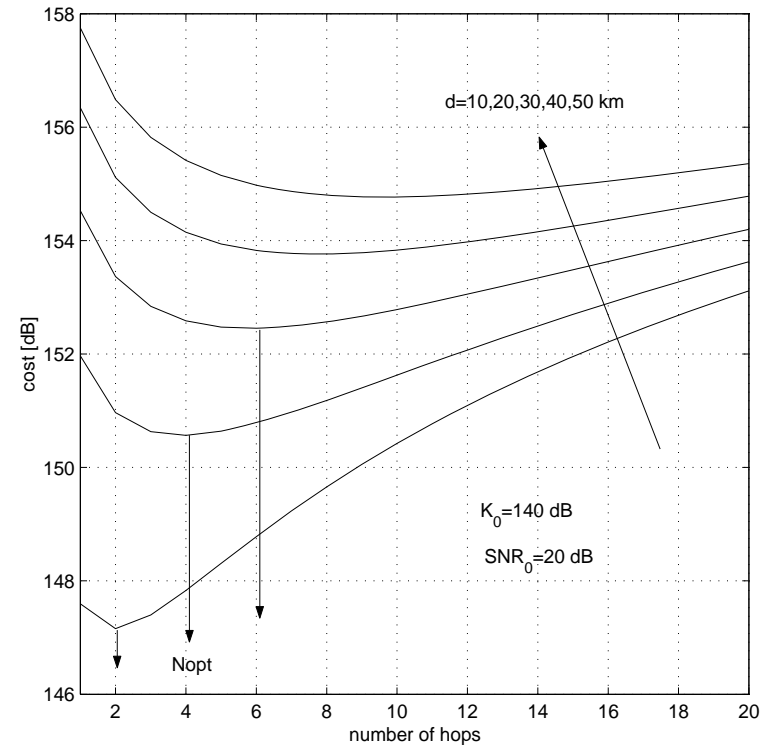

Fig. 11. Cost function based on the total power consumption as defined by the expression (6). Different curves correspond to different link distances $d$.

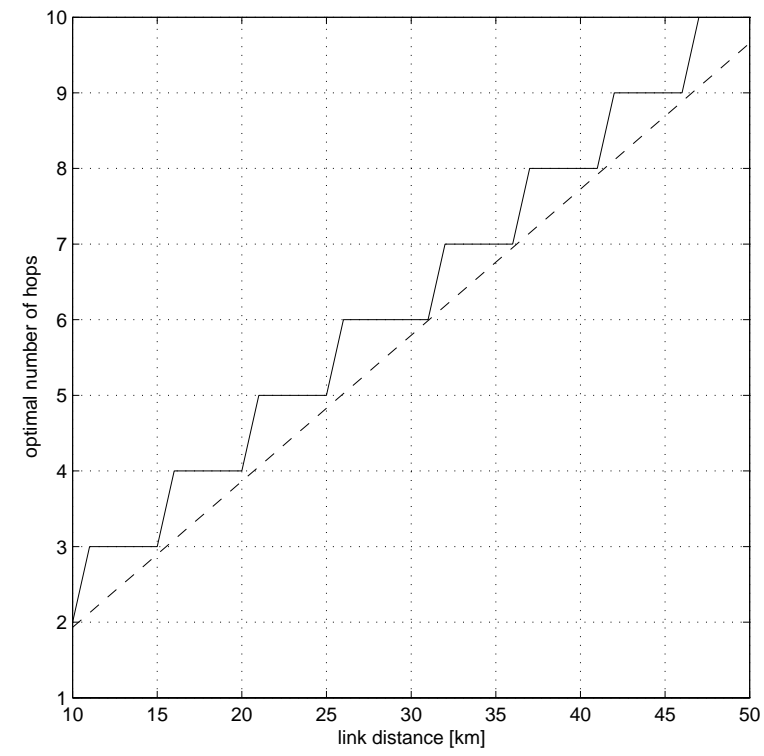

Fig. 12. Optimal number of hops corresponding to the cost function of Fig.11. Dashed line shows the continuous value (7).

In the second case, the transmission rate is allowed to vary with the hop distance, so that it can be optimized according to the link capacity. In this case, the total delay is no longer a simple linear function of the number of relays. Instead, it depends on the number of hops through the transmission rate $R$.

In a practical system, the transmission rate will be proportional to the available link bandwidth, which we have seen to depend on the distance $d / N$. Ideally, from an informationtheoretic point of view, error-free transmission can be accomplished at the rate equal to the channel capacity. In either case, the available transmission rate becomes a function of the

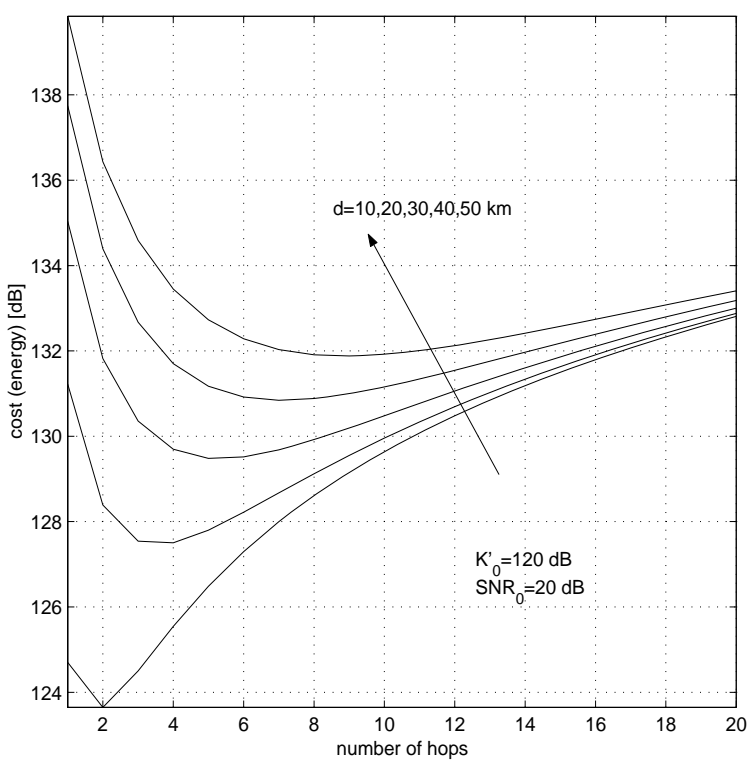

Fig. 13. Cost function based on the energy per bit as defined by the expression (8). Different curves correspond to different link distances $d$.

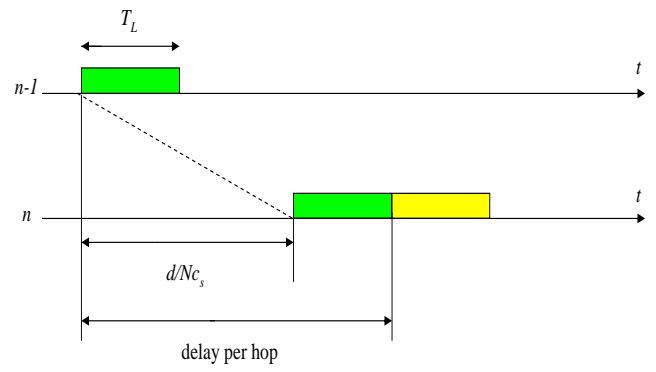

Fig. 14. Delay between relays.

number of hops $N$. By increasing the number of hops, more packets contribute to the overall delay by their duration $T_{L}$, but each packet duration becomes shorter because the transmission can be accomplished faster over a shorter hop. Assuming that the transmission is accomplished at the rate equal to the link capacity, we obtain the total delay as

$$
D_{N}(d)=\frac{d}{c_{s}}+\frac{L}{C(d / N)} N=\frac{d}{c_{s}}+\frac{d^{\gamma} L}{c} N^{1-\gamma}
$$

The delay still increases with the number of hops since $\gamma<1$ (see Fig.2). However, it does so at a lower rate as compared to the fixed rate delay (10), $1-\gamma<1$. 
As an example, let us consider transmission of a packet consisting of $L=5000$ bits. When the rate is fixed, its value is set to $R=2000 \mathrm{bits} / \mathrm{s} .{ }^{2}$ Fig. 15 illustrates the total delay as a function of the number of hops. Two sets of curves are included: the delay corresponding to the fixed transmission rate is shown in dashed line, while the delay corresponding to the optimally adjusted transmission rate is shown in solid and in dash-dot line. The solid curves correspond to the rate equal to the channel capacity, $R=C(d / N)$. The dash-dot curves show the delay obtained with the rate equal to the channel bandwidth, $R=B(d / N)$.

The result of Fig.15 reveals that the delay penalty can be kept at a minimum by adjusting the rate in accordance with the hop distance. While the delay corresponding to the fixed rate increases linearly with the number of relays, the delay corresponding to the optimal rate adjustment appears almost constant. Moreover, the difference in delay between transmission at a rate equal to the bandwidth (which is a practically viable solution with coding techniques available for underwater acoustic channels) and transmission at a rate equal to the channel capacity is negligible. Hence, if the delay is the figure of merit for a particular system design, rate adjustment in accordance with the distance will keep this cost at a minimum. The number of hops can then be chosen so as to satisfy another system requirement, such as power or energy minimization. We note that this property is specific to an acoustic environment, as it stems from the relationship between the link capacity (or bandwidth) and the transmission distance.

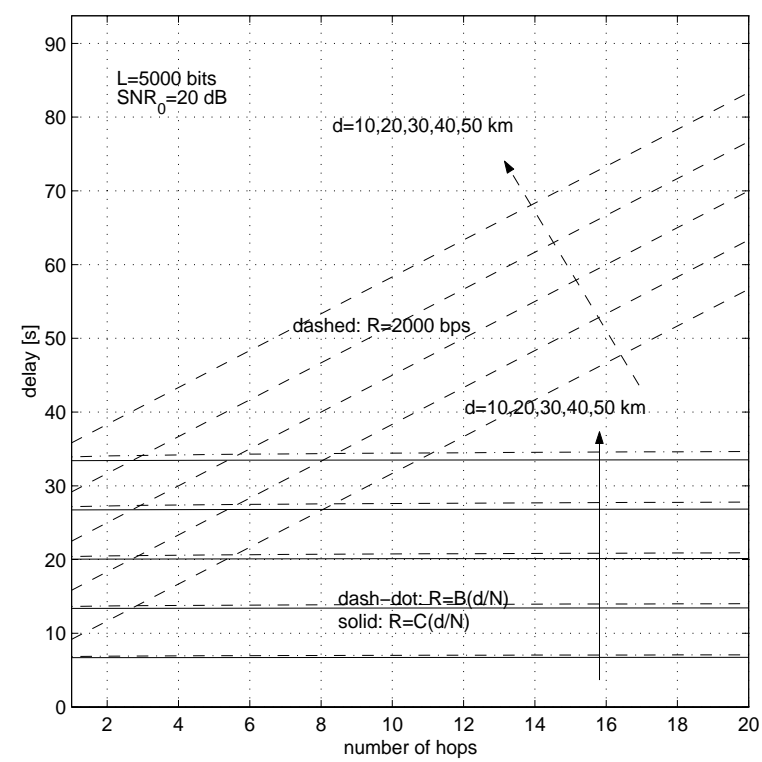

Fig. 15. Delay of the relay channel as a function of the number of hops $N$ for varying distance.

\footnotetext{
${ }^{2}$ The packet size of 5000 bits would be an optimal choice for transmission at this rate over a $15 \mathrm{~km}$ link characterized by a BER of $10^{-4}$, if a selective Stop \& Wait ARQ protocol were used with groups of 8 packets [3].
}

\section{CONCLUSION}

A relay acoustic link may be needed in a number of applications where data transmission has to be accomplished beyond short distances. Since the available acoustic bandwidth decays with distance, it is advantageous to accomplish such transmission using relays. To assess the trade-offs involved in the deign of a relay acoustic link, we have addressed the link capacity, as well as the associated issues of power and energy consumption, system cost, and link delay.

A mathematical model that relates the capacity of a link to its distance was employed, showing that the capacity increases with the number of relays. Specifically, the capacity of a link with $N$ hops behaves as $N^{\gamma}$, where $\gamma$ is a positive constant, whose exact value depends both on the channel and the SNR (for a channel modeled by the basic acoustic propagation loss, practical spreading, and a typical ambient noise spectrum, $\gamma$ was found to vary between 0.4 and 0.8 for a wide range of SNRs). Accompanying the capacity increase with the number of relays is a reduction in the power required to span the link. However, each relay increases the overall system cost, and this fact must be taken into account when designing a system. System optimization was addressed in light of minimizing a cost function, defined as the total power needed to span the link (or the required energy per bit) plus a fixed cost of each relay. Minimization of the cost function provides an analytical solution for the optimal number of relays.

Another form of assessing the cost of relaying is through the overall link delay. As each relay introduces a delay proportional to the length of the data packet transmitted, the link delay depends on the number of relays. However, it also depends on the rate at which the information is transmitted, which determines the duration of a data packet. This fact implies an interesting property of an acoustic link: although each relay introduces an additional delay, as the hops become shorter transmission can be accomplished faster over each hop. As a result, the delay does not increase linearly with the number of hops, as it would if the transmission rate were kept constant irrespective of the number of relays, but at a (much) slower rate. In particular, if transmission is accomplished at the rate equal to the link capacity, the overall delay grows as $N^{1-\gamma}$. This improvement is specific to the underwater acoustic environment, where the channel capacity and bandwidth are dependent on the transmission distance.

\section{ACKNOWLEDGMENT}

This work was supported by the following grants: ONR MURI Grant \# N00014-07-1-0738, NOAA Sea Grant NA060AR4170019, NSF grants 0520075 and 0427502 .

\section{REFERENCES}

[1] M.Stojanovic, "On the relationship between capacity and distance in an underwater acoustic channel," in Proc. First ACM International Workshop on Underwater Networks (WUWNet), Sept. 2006.

[2] J.Partan, J.Kurose and B.N.Levine, "A Survey of practical issues in underwater networks," in Proc. First ACM International Workshop on Underwater Networks (WUWNet), Sept. 2006.

[3] M.Stojanovic, "Optimization of a data link protocol for an underwater acoustic channel," in Proc. IEEE Oceans'05 Europe Conf., June 2005. 